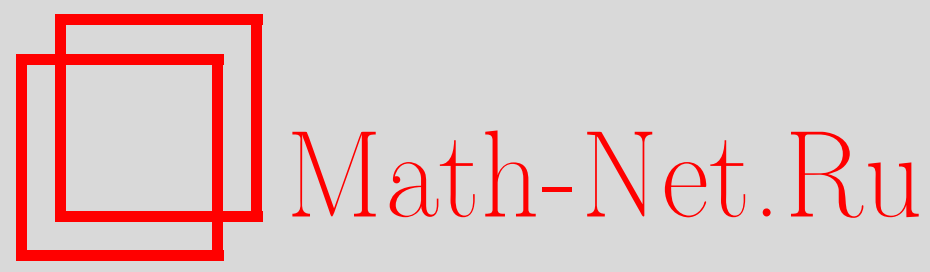

Г. Г. Аманжаев, О сложности приближенного табличного задания дискретных аналогов функций конечной гладкости в метрике $L^{p}$, Матем. заметки, 1998, том 64, выпуск 5, 643647

DOI: https://doi.org/10.4213/mzm1440

Использование Общероссийского математического портала Math-Net.Ru подразумевает, что вы прочитали и согласны с пользовательским соглашением http://www.mathnet.ru/rus/agreement

Параметры загрузки:

IP: 54.166 .219 .16

26 апреля 2023 г., 13:07:35

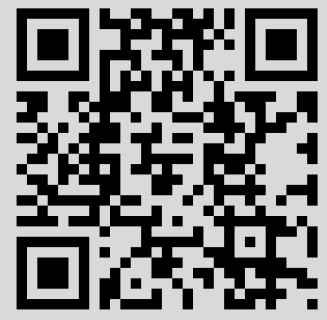


том 64 выпуск 5 нояБРь 1998

УДК $517.5+519.7$

\section{О СЛОЖНОСТИ ПРИБЛИЖЕННОГО ТАБЛИЧНОГО ЗАДАНИЯ ДИСКРЕТНЫХ АНАЛОГОВ ФУНКЦИЙ КОНЕЧНОЙ ГЛАДКОСТИ В МЕТРИКЕ $L^{p}$ \\ Г. Г. Аманжаев}

Для дискретњых аналогов классов функций конечной гладкости исследовалась величина log Approx, характеризующая минимально необходимую длину таблиц, позволяющих восстанавливать функции из этих классов с погрешностью не более 1 в метрике пространства $L^{p}$.

Библиография: 5 названий.

В работах [1]-[3] были определены различные классы функций, являющиеся дискретными аналогами классов функций конечной гладкости, и исследовалась величина $\log$ Approx, характеризующая минимально необходимую длину таблищ, позволяющих восстанавливать функции из этих классов с погрешностью не более 1 . В настоящей работе аналогичные результаты (анонсированные в указанных работах) доказаны для случая приближений в других метриках, а именно, в метрике пространства функций вида $f:\{0,1, \ldots, N-1\} \rightarrow \mathbb{R}$ с нормой

$$
\|f\|_{p}=\left(\frac{1}{N} \sum_{i=0}^{N-1}|f(i)|^{p}\right)^{1 / p}
$$

(нормирующий множитель $1 / N$ выбран так, чтобы нормой константы была сама эта константа). Обозначая также равномерную норму

$$
\|f\|=\max _{x}|f(x)|=\lim _{p \rightarrow \infty}\|f\|_{p}
$$

через $\|f\|_{\infty}$, имеем для любой функции $f$ и любых $p, q, 1 \leqslant p \leqslant q \leqslant+\infty$, соотношение

$$
\|f\|_{1} \leqslant\|f\|_{p} \leqslant\|f\|_{q} \leqslant\|f\|_{\infty}=\|f\|
$$

причем для $f=$ const все эти неравенства обращаются в равенства.

Будем говорить, что множество дискретных функций $A$ вида $f:\{0,1, \ldots, N-1\} \rightarrow$

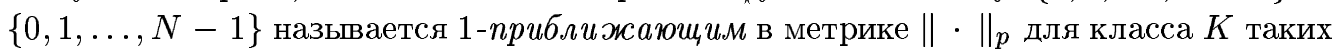

Работа выполнена при частичной финансовой поддержке Российского фонда фундаментальнх исследований, грант № 96-01-01068. 
функций, если для каждой функции $f \in K$ в $A$ найдется такая функция $g$, для которой $\|f-g\|_{p} \leqslant 1$.

Обозначим минимальную мощность 1-приближающего в метрике $\|\cdot\|_{p}$ множества для класса $K$ через $\operatorname{Approx}_{p} K$.

Связь между такими нормами для разных $p$ приводит при $1 \leqslant p \leqslant q \leqslant \infty$ к неравенству

$$
\operatorname{Approx}_{1} K \leqslant \operatorname{Approx}_{p} K \leqslant \operatorname{Approx}_{q} K \leqslant \operatorname{Approx}_{\infty} K .
$$

Из этого неравенства видно, что верхняя оценка для $\mathrm{Approx}_{\infty} K$ является верхней оценкой всех $\operatorname{Approx}_{p} K$, а для получения нижней достаточно исследовать только случай метрики $\|\cdot\|_{1}$ (конечно, если эти нижняя и верхняя оценки окажутся одного порядка).

Наша задача - нахождениепорядка роста (при $N \rightarrow \infty$ ) логарифма этих величин для дискретных аналогов классов функций конечной гладкости. Проведем соответствующий анализ на примере дискретных аналогов классов $H_{n, \omega}$ при $n \geqslant 1$, которые заданы следуюшим образом. Пусть $H_{n, \omega}$ - это множество функций вида $f:[0,1) \rightarrow[0,1)$, имеющих $n$ производных, причем для $n$-й производной модуль непрерывности мажорируется функцией $\omega$. Внешним дискретным аналогом класса $H_{n, \omega}$ при $N$ уровнях квантования назовем класс дискретных функций

$$
\begin{aligned}
\widehat{H}_{n, \omega}^{N}= & \{g:\{0,1, \ldots, N-1\} \rightarrow\{0,1, \ldots, N-1\} \mid \\
& \left.\exists f \in H_{n, \omega} \quad \forall x \quad g(x)=\left\lfloor N f\left(\frac{x}{N}+\frac{1}{2 N}\right)\right\rfloor\right\} .
\end{aligned}
$$

Для функций из $\widehat{H}_{n, \omega}$ имеет место оценка конечных разностей:

$$
\left|\Delta_{n+1}\left(g ; x_{0}, \ldots, x_{n+1}\right)\right|<\frac{\omega\left(\left(x_{n+1}-x_{0}\right) / N\right)}{\left(x_{n+1}-x_{0}\right) n ! N^{n-1}}+\varphi_{n+1}\left(x_{0}, \ldots, x_{n+1}\right),
$$

где через $\Delta_{n+1}\left(g ; x_{0}, \ldots, x_{n+1}\right)$ обозначена разделенная конечная разность (см. [4]) $(n+1)$-го порядка с узлами $x_{0}, \ldots, x_{n+1}$ от функции $g$, а величина

$$
\varphi_{n}\left(x_{0}, \ldots, x_{n+1}\right)=\max _{|h| \leqslant 1 / 2} \Delta_{n+1}\left(h ; x_{0}, \ldots, x_{n+1}\right),
$$

где максимум берется по всем функциям $h$, не превьшающим $1 / 2$ по абсолютной величине.

Внутренним дискретным аналогом $H_{n, \omega}^{N}$ класса $H_{n, \omega}$ при $N$ уровнях квантования назовем класс дискретных функций вида $g:\{0,1, \ldots, N-1\} \rightarrow\{0,1, \ldots, N-1\}$, удовлетворяющих неравенству (2) при любом наборе различных $x_{0}, \ldots, x_{n+1} \in\{0,1, \ldots, N-1\}$.

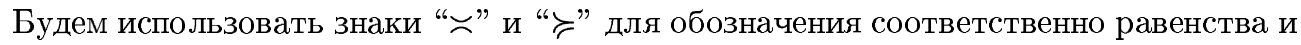
(нестрогого) неравенства по порядку.

Основным результатом настоящей работы является

ТЕОРема. Пусть $\omega-$ выпуклая вверх функция типа модуля непрерывности, $n \geqslant 1,1 \leqslant p \leqslant+\infty$. Тогда при $N \rightarrow \infty$ имеет место соотноиение

$$
\log \operatorname{Approx}_{p} \widehat{H}_{n, \omega}^{N} \asymp \log \operatorname{Approx}_{p} H_{n, \omega}^{N} \asymp \frac{1}{W(1 / N)},
$$


где $W-$ функиия, обратная $\kappa t^{n} \omega(t)$.

С учетом того, что в [1]-[3] эта оценка была доказана при $p=+\infty$, а также неравенств (1) и очевидного соотношения $\operatorname{Approx}_{p} \widehat{H}_{n, \omega}^{N} \leqslant \operatorname{Approx}_{p} H_{n, \omega}^{N}$ для доказательства этой теоремы не хватает только соотношения

$$
\log \operatorname{Approx}_{p} \widehat{H}_{n, \omega}^{N} \succcurlyeq \frac{1}{W(1 / N)} .
$$

Нам потребуется одно комбинаторное утверждение.

Лемма. При $\alpha \in(0,1 / 4), n \rightarrow \infty, m \sim \alpha$ множество двоичных наборов длины $n$ содержит подмножество мощности не меньше

$$
2^{n(1+2 \alpha \log (2 \alpha)+(1-2 \alpha) \log (1-2 \alpha)+o(1))}
$$

(логарифм этой величины имеет порядок $n$ ), әлементы которого попарно отличаются не менее чем в $т$ разрядах.

ДокаЗАТЕльство. Рассмотрим намножествевсех двоичных наборов длины $n$ с метрикой Хемминга шар радиуса $r$ с центром $a$, где $a=\left(a_{1}, \ldots, a_{n}\right), a_{i} \in\{0,1\}$. Пусть $V(r)$ - объем такого шара (число всех его точек). Тогда (см., например, [5, c. 312]) мощность рассматриваемого множества не меньше чем $x=2^{n} / V(2 m-1)$. Оценим эту величину снизу, учитывая то, что $V(r)=C_{n}^{0}+C_{n}^{1}+\cdots+C_{n}^{r}$.

Итак,

$$
x=\frac{2^{n}}{C_{n}^{0}+C_{n}^{1}+\cdots+C_{n}^{2 m-1}} .
$$

Среди этих $2 m$ слагаемых при достаточно больших $n$ наибольшим будет последнее. Поэтому $x \geqslant 2^{n} /\left(2 m C_{n}^{2 m}\right)$, т.е. $\log x \geqslant n-\log (2 m)-\log C_{n}^{2 m}$. По формуле Стирлинга $k ! \sim \sqrt{2 \pi k}(k / e)^{k}$ имеем

$$
\begin{aligned}
\log C_{n}^{2 m} & =\log \frac{n !}{(2 m !)(n-2 m) !}=\log \frac{\sqrt{2 \pi n}(n / e)^{n}(1+o(1))}{\sqrt{4 \pi m}(2 m / e)^{2 m} \sqrt{2 \pi(n-2 m)}((n-2 m) / e)^{n-2 m}} \\
& =\log \sqrt{\frac{n}{4 \pi m(n-2 m)}}+2 m \log \frac{n}{2 m}+(n-2 m) \log \frac{n}{n-2 m}+o(1) \\
& =n\left(2 \alpha \log \frac{1}{2 \alpha}+(1-2 \alpha) \log \frac{1}{1-2 \alpha}+o(1)\right) .
\end{aligned}
$$

Поэтому $\log x \geqslant n(1+2 \alpha \log (2 \alpha)+(1-2 \alpha) \log (1-2 \alpha)+o(1))$. Покажем, что последняя величина имеет порядок $n$, т.е. что

$$
1+2 \alpha \log 2 \alpha+(1-2 \alpha) \log (1-2 \alpha)>0 .
$$

Обозначим $\beta=4 \alpha-1$ (при этом $-1<\beta<0$ ). Тогда неравенство (3) примет вид

$$
1+\frac{1+\beta}{2} \log \frac{1+\beta}{2}+\frac{1-\beta}{2} \log \frac{1-\beta}{2}>0,
$$

или после преобразований

$$
(1+\beta) \ln (1+\beta)+(1-\beta) \ln (1-\beta)>0 .
$$

Как легко проверить дифференцированием, левая часть этого неравенства минимальна при $\beta=0$, а при $\beta \neq 0$ она положительна. Тем самым, лемма доказана. 
ДОКАЗАТЕЛЬСТво ТЕОРЕМЫ. Рассмотрим множество функций, имеющих вид

$$
f(x)=\sum_{l=0}^{\left\lfloor 1 /\left(2^{n+1} \varepsilon\right)\right\rfloor-1} a_{l} \delta_{n, \varepsilon}\left(x-2^{n+1} l \varepsilon\right),
$$

где $a_{l} \in\{0,1\}$, а функции $\delta_{n, \varepsilon}$ определены следующим образом:

$$
\begin{aligned}
& \delta_{0, \varepsilon}(x)= \begin{cases}0, & x \leqslant 0, \\
\omega(x) / 2, & 0 \leqslant x \leqslant \varepsilon, \\
\omega(2 \varepsilon-x) / 2, & \varepsilon \leqslant x \leqslant 2 \varepsilon, \\
0, & x \geqslant 2 \varepsilon,\end{cases} \\
& \delta_{1, \varepsilon}(x)=\int_{0}^{x}\left(\delta_{0, \varepsilon}(t)-\delta_{0, \varepsilon}(t-2 \varepsilon)\right) d t, \\
& \delta_{2, \varepsilon}(x)=\int_{0}^{x}\left(\delta_{1, \varepsilon}(t)-\delta_{1, \varepsilon}(t-4 \varepsilon)\right) d t, \\
& \delta_{n, \varepsilon}(x)=\int_{0}^{x}\left(\delta_{n-1, \varepsilon}(t)-\delta_{n-1, \varepsilon}\left(t-2^{n} \varepsilon\right)\right) d t,
\end{aligned}
$$

т.е. $\delta_{n, \varepsilon}$ имеет вид неотрицательной “шапочки” с носителем $\left[0,2^{n+1} \varepsilon\right], n$ раз дифференцируема, причем модуль непрерывности $n$-й производной мажорируется функцией $\omega / 2$, а также при $\varepsilon \rightarrow 0$ вьполнены соотношения

$$
\max _{x} \delta_{n, \varepsilon}(x) \asymp \varepsilon^{n} \omega(\varepsilon), \quad \int_{-\infty}^{\infty} \delta_{n, \varepsilon}(x) d x \asymp \varepsilon^{n+1} \omega(\varepsilon) .
$$

Пусть $f_{1}$ и $f_{2}$ - две функции вида (4), заданные соответственно наборами коэффициентов $a^{\prime}=\left(a_{0}^{\prime}, a_{1}^{\prime}, \ldots\right)$ и $a^{\prime \prime}=\left(a_{0}^{\prime \prime}, a_{1}^{\prime \prime}, \ldots\right)$. Оценим снизу величину $\left\|\widehat{f}_{1}-\widehat{f}_{2}\right\|_{1}$ через $\sum_{i}\left|a_{i}^{\prime}-a_{i}^{\prime \prime}\right|$, где $\widehat{f}:\{0,1, \ldots, N-1\} \rightarrow\{0,1, \ldots, N-1\}$ по определению есть $\lfloor N f(x / N+1 /(2 N))\rfloor$. В силу того, что носители разных функций $\delta$ (при разных $l$ ) не имеют обших внутренних точек, имеем

$$
\left\|\widehat{f}_{1}-\widehat{f}_{2}\right\|_{1}=\sum_{l}\left|a_{l}^{\prime}-a_{l}^{\prime \prime}\right| \cdot\left\|\widehat{\delta}_{n, \varepsilon}\left(\cdot-2^{n+1} l \varepsilon\right)\right\|_{1} \geqslant \min _{t}\left\|\widehat{\delta}_{n, \varepsilon}(\cdot-t)\right\|_{1} \sum_{l}\left|a_{l}^{\prime}-a_{l}^{\prime \prime}\right| .
$$

Далее, $\widehat{\delta}_{n, \varepsilon}$ есть функция вида $\left\lfloor N \delta_{n, \varepsilon}(x / N+\right.$ const $\left.)\right\rfloor$, т.е.

$$
\min _{t}\left\|\widehat{\delta}_{n, \varepsilon}(\cdot-t)\right\|_{1}=\min _{u}\left\|\left\lfloor N \delta_{n, \varepsilon}(\cdot / N-u)\right\rfloor\right\|_{1} \geqslant \min _{u} \sum_{x \in \mathbb{Z}} \delta_{n, \varepsilon}\left(\frac{x}{N}-u\right)-2^{n+1} N \varepsilon .
$$

Функции $\delta$ неотрицательны и имеют один максимум; поэтому

$$
\begin{aligned}
\sum_{x \in \mathbb{Z}} \delta_{n, \varepsilon}\left(\frac{x}{N}-u\right) & \geqslant \int_{-\infty}^{\infty} \delta_{n, \varepsilon}\left(\frac{x}{N}-u\right) d x-\max _{t} \delta_{n, \varepsilon}(t) \\
& =N \int_{-\infty}^{\infty} \delta_{n, \varepsilon}(t) d t-\max _{t} \delta_{n, \varepsilon}(t) \succcurlyeq \varepsilon^{n} \omega(\varepsilon)\left(c_{1} N \varepsilon-c_{2}\right),
\end{aligned}
$$


где $c_{1}, c_{2}>0$. Поэтому при $\varepsilon \gg 1 / N$ имеем

$$
\left\|\widehat{f}_{1}-\widehat{f}_{2}\right\|_{1} \succcurlyeq N \varepsilon^{n+1} \omega(\varepsilon) \sum_{l}\left|a_{l}^{\prime}-a_{l}^{\prime \prime}\right|-2^{n+1} N \varepsilon .
$$

Если теперь

$$
\sum_{l}\left|a_{l}^{\prime}-a_{l}^{\prime \prime}\right| \geqslant c \sum_{l} 1 \sim \frac{c}{2^{n+1} \varepsilon}
$$

где $c=$ const $>0$, то

$$
\left\|\widehat{f}_{1}-\widehat{f}_{2}\right\|_{1} \succcurlyeq N\left(c c_{3} \varepsilon^{n} \omega(\varepsilon)-\varepsilon c_{4}\right)
$$

где $c_{3}, c_{4}>0$.

Выбирая $\varepsilon$ вида $W\left(c_{5} / N\right)$, где $c_{5}=$ const, получаем

$$
\left\|\widehat{f}_{1}-\widehat{f}_{2}\right\|_{1} \succcurlyeq c c_{3} c_{5}-c_{4} N W\left(\frac{c_{5}}{N}\right) .
$$

По построению $N W(N) \rightarrow \infty$ при $N \rightarrow \infty$. Поэтому выбором константы $c_{5}$ можно добиться того, что при достаточно больших $N$ будет вьполнено неравенство $\left\|\widehat{f}_{1}-\widehat{f}_{2}\right\|_{1} \geqslant 3$.

Тем самым, для построения 3-различимого (в метрике $\|\cdot\|_{1}$ ) множества функций надо уметь подбирать соответствующие наборы коэффициентов $\left(a_{0}, a_{1}, \ldots\right)$ длины $d=$ $\left[1 /\left(2^{n+1} \varepsilon\right)\right]$, различающиеся не менее чем в $c d$ разрядах, для какой-то положительной константы $c$; при этом число таких наборов будет равно мощности соответствующего 3-различимого множества. Это можно сделать по доказанной лемме: для любого достаточно малого $c$ соответствующая мощность будет не меньше $2^{c_{6}} d$, где $c_{6}=c_{6}(c)-$ положительная константа.

Итак, построено 3-различимое (в метрике $\|\cdot\|_{1}$ ) множество мощности $2^{\text {const } / \varepsilon}$. Из выбора $\varepsilon$ имеем

$$
\log \operatorname{Approx}_{1} \widehat{H}_{n, \omega}^{N} \succcurlyeq \frac{1}{\varepsilon} \asymp \frac{1}{W(1 / N)} .
$$

Теорема доказана.

Автор выражает глубокую благодарность своему руководителю О. Б. Лупанову за постановку задач и постоянное внимание к работе.

\section{СПИСОК ЦИТИРОВАННОЙ ЛИТЕРАТУРЫ}

[1] Аманжаев Г. Г. О дискретных аналогах классов непрерывных функций различной гладкости // Докл. РАН. 1995. Т. 342. № 2. С. 54-58.

[2] Аманжаев Г. Г. О дискретных аналогах классов функций, задаваемых модулем непрерывности $n$-й производной // Вестн. МГУ. Сер. 1. Матем., мех. 1996. № 2. С. 3-8.

[3] Аманжаев Г. Г. О дискретных аналогах функций дробной гладкости // Вестн. МГУ. Сер. 1. Матем., мех. 1996. № 4. С. 3-7.

[4] Гельфонд А. О. Исчисление конечных разностей. М.: Наука, 1967.

[5] Яблонский С.В., Лупанов О.Б. (ред. ) Дискретная математика и математические вопросы кибернетики. Т. 1. М.: Наука, 1974. 